\begin{tabular}{|l|l|l||}
\hline \multicolumn{2}{|c|}{ PublisherInfo } \\
\hline \hline PublisherName & $:$ & BioMed Central \\
\hline \hline PublisherLocation & $:$ & London \\
\hline \hline PublisherImprintName & $:$ & BioMed Central \\
\hline \hline
\end{tabular}

\title{
Influence of cardiopulmonary resuscitation prior to defibrillation in patients with out-of-hospital ventricular fibrillation
}

\begin{tabular}{|l|l|l||}
\hline \multicolumn{2}{|c||}{ ArticleInfo } \\
\hline \hline ArticleID & $:$ & 4082 \\
\hline \hline ArticleDOI & $:$ & $10.1186 /$ ccf-1999-124 \\
\hline \hline ArticleCitationID & $:$ & 124 \\
\hline \hline ArticleSequenceNumber & $:$ & 19 \\
\hline \hline ArticleCategory & $:$ & Paper Report \\
\hline \hline ArticleFirstPage & $:$ & 1 \\
\hline \hline ArticleLastPage & $:$ & 4 \\
\hline \hline & & RegistrationDate : 1999-5-7 \\
ArticleHistory & $:$ & OnlineDate $\quad$ 1999-5-7 \\
\hline \hline ArticleCopyright & $:$ & Current Science Ltd1999 \\
\hline \hline ArticleGrants & $:$ & \\
\hline \hline ArticleContext & $:$ & 130541111 \\
\hline \hline
\end{tabular}




\section{Keywords}

Cardiopulmonary resuscitation, defibrillation, ventricular fibrillation

\section{Comments}

The results of this study are in some ways unsurprising. There can be no doubt that without defibrillation VF is not survivable, but the results presented serve to illustrate the importance of basic life support, as without adequate perfusion and oxygenation, as supplied by CPR, survival is not possible. Once the clock has gone beyond three or four min the priorities clearly change as hypoxic brain damage will ensue and delaying CPR to administer shocks does the patient no favours. Clearly this applies mainly to out of hospital arrests where delay is inherent. Clinical studies of cardiac arrest are fraught with difficulties and advances have been few and slow. This study should be welcomed therefore and it seems likely that the effect demonstrated is a true one. This was an observational study.

\section{Introduction}

The definitive treatment for ventricular fibrillation (VF) is defibrillation and the speed with which this can be applied is an important factor in determining survival for victims of out of hospital VF. Since 1980 in Seattle, Washington, early defibrillation has been employed by first responding emergency units (often fire dept. personnel), using automated external defibrillators. Despite this, and the consequent significant reduction in time (by three to four min) to first shock, overall survival rates for victims of out of hospital VF remained unchanged, although still very impresive at around 20\%. Pre 1994 immediate defibrillation had been the priority of first responders, at the expense of initial basic life support.

Experimental animal work has demonstrated that CPR and adrenaline prior to defibrillation (for VF of 7.5 min duration) improves survival rates. This, and the lack of survival benefit from apparent early defibrillation in out of hospital VF, prompted a change in the priorities of first attending emergency medical personnel in Seattle.

\section{Aims}


To assess whether 90 seconds of basic life support prior to first defibrillation would result in a survival benefit for victims of out of hospital VF.

\section{Methods}

Externally controlled or observational study. The outcome for 639 patients treated during the 42 months before the policy change in resuscitation (01/01/94) was compared to that of 478 patients treated in the 36 months after the change. Demographic variables for the two groups were very similar. The main outcome measures were survival and degree of neurological recovery at time of discharge, as established by review of hospital notes and as a function of response times ( $<$ four min or $>$ four min). Neurological recovery was graded 1 to 4 , level 1 suggesting full/near full recovery and level 4 being consistent with a persistent vegetative state or similar. Level 1 and 2 patients were considered to have made a favourable early neurological recovery.

\section{Results}

Overall survival was improved from $24 \%$ to $30 \%(P=0.4)$ following the institution of 90 seconds of CPR before defibrillation. This was most noticeable when the response time was more than 4 minutes ( $17 \%$ versus $27 \% ; P<0.007)$. Using multivariate logistic regression analysis, adjusting for covariables affecting survival between the two time periods, the change in resuscitation protocol was found to significantly improve survival (odds ratio $1.42 ; 95 \%$ confidence interval $1.07-1.90 ; P=0.2$ ). Patients surviving with a favourable neurological outcome improved from $17 \%$ to $23 \%(P=0.1)$.

\section{Discussion}

There was a significant improvement in survival for out of hospital VF in the second time period studied. The authors comment that while the resuscitation protocol for first attending emergency personnel was clearly changed during this time they cannot conclusively exclude other causes for the findings. But survival rates had been static/declining prior to the protocol change. However it is commented that for 25 months of the second time period studied a concurrent randomized trial of amiodarone $v s$ placebo was performed in a subgroup of patients. It was felt that this was unlikely to have affected the outcome, however, as only $16 \%$ of patients received amiodarone and no survival benefit from its use was seen. It is noted that a distinction must be made between hospitalized, monitored patients developing VF and receiving very rapid defibrillation, where excellent outcomes are found, and out of hospital victims where there is inevitably a delay of several minutes. Improving oxygenation, brain perfusion, metabolic variables etc. would seem to be important in this group. Attention is drawn to the probable detrimental effects of applying immediate automated defibrillation to patients with asystole 
or electromechanical disociation, where immediate CPR is needed. The authors feel their findings represent an important pilot study and that randomized trials be conducted to further quantify the benefit of CPR before defibrillation, where delay has been encountered.

\section{References}

1. Cobb LA, Fahrenbruch CE, Walsh TR, et al: Influence of cardiopulmonary resuscitation prior to defibrillation in patients with out-of-hospital ventricular fibrillation. JAMA. 1999, 281: 1182-1188.

This PDF file was created after publication. 